\title{
Advanced hydroxychloroquine maculopathy against medical advice
}

\author{
Michael N. Cohen, Bryan K. Hong, Richard S. Kaiser \\ Mid Atlantic Retina, The Retina Service of Wills Eye Hospital, Thomas J efferson University, Philadelphia, Pennsylvania, USA \\ Correspondence: Richard S. Kaiser. Address: Mid Atlantic Retina, The Retina Service of Wills Eye Hospital, Thomas \\ Jefferson University, Philadelphia, Pennsylvania 19107, USA. Email: RKaiser@midatlanticretina.com \\ Received: J une 23, 2015 \\ DOI : $10.5430 /$ crim.v2n3p52 \\ Accepted: July 09, 2015 \\ Online Published: July 24, 2015
}

\section{Abstract}

A 67-year-old female with systemic lupus erythematosus returned to her ophthalmologist for evaluation of nyctalopia and decreased peripheral vision in both eyes for several months. On her initial presentation two years prior, she was noted to have signs of advanced hydroxychloroquine toxicity on fundus examination, fundus autofluorescence, and optical coherence tomography. Despite counseling by multiple physicians, she refused to discontinue the medication and did not return for her regularly scheduled eye examinations for the next 24 months. Her current presentation details and documents (with several imaging modalities) the progression of advanced hydroxychloroquine maculopathy while continuing treatment. With some regularity, Ophthalmologists are faced with the difficult clinical dilemma to help determine if a patient should discontinue HCQ therapy. This case emphasizes why detecting clinical toxicity and discontinuing therapy is crucial for the safe usage of this frequently prescribed medication.

\section{Keywords}

Hydroxychloroquine, Ophthalmology, Retina, Maculopathy, Toxicity, Plaquenil, Systemic lupus erythematosus, Screening

\section{Presentation}

A 67-year-old female with hypertension, systemic lupus erythematosus, and rheumatoid arthritis, presented for evaluation of nyctalopia and decreased peripheral vision in both eyes for several months. She was taking $400 \mathrm{mg}$ of hydroxychloroquine (HCQ) daily and had been on HCQ treatment for over a decade. On her initial presentation in 2012, she was noted to have signs of HCQ toxicity on fundus examination, fundus autofluorescence, and optical coherence tomography (see Figures 1 and 2). At the urging of her ophthalmologist she stopped HCQ, but only for a few months. She soon decided, under her own volition, to re-start the medication, which she has now been taking against medical advice for over fifteen months. She did not return for her regularly scheduled eye examinations. The remainder of her medical history is as follows:

- $\quad$ Past Medical History: Hypertension, Systemic Lupus Erythematosis, and Rheumatoid arthritis;

- $\quad$ Past Surgical History: Cataract Extraction with Intraocular Lens Implantaion, Left Eye;

- $\quad$ Family History: Coronary Artery Disease (father), Hypertention (father); 
- $\quad$ Social History: Non-smoker. No alcohol use. No illicit drug use.

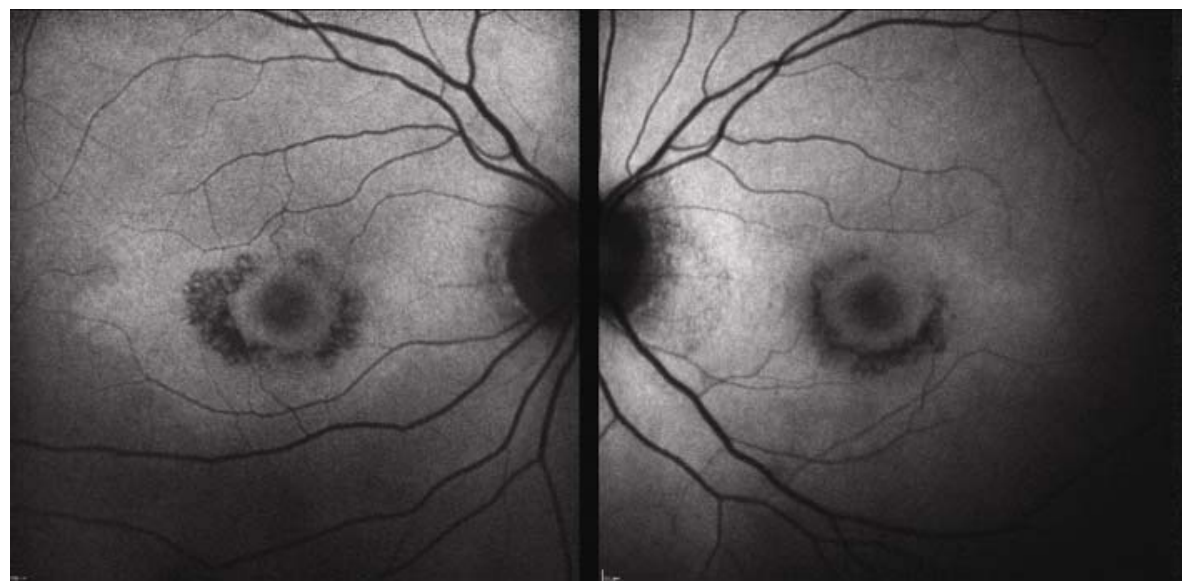

Figure 1. Fundus Autofluorescence of the right (left-most image) and the left (right-most image) eye from 2012. The dark areas of hypo-autofluorescence represent sick or damaged RPE cells in the macula, located in a bull's-eye pattern around the fovea

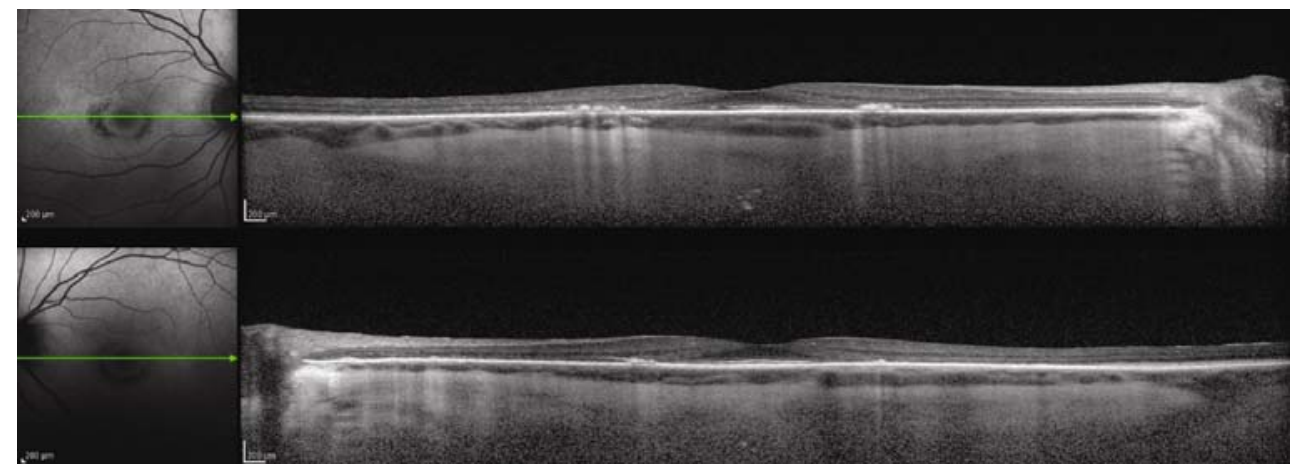

Figure 2. Optical Coherence Tomography of the right (upper image) and the left (lower image) eye from 2012 demonstrates retinal layer disruption, highlighted by a loss of the external limiting membrane, disruption of the outer ellipsoid zone, RPE granularity, and progressive parafoveal thinning of the all retinal layers. Note the "flying saucer sign", representing the presumed sparing of the sub-foveal ellipsoid layer

\section{Examination}

In 2014, she finally returned for an ophthalmic evaluation. General vital signs from her recent primary care appointment were notable for a heart rate of 87 beats/min and a blood pressure of 134/77. Recent systemic examination and laboratory work was within normal limits. Ophthalmic examination revealed a visual acuity of 20/50 in the right eye and 20/30 in the left eye. Both pupils were reactive with no afferent pupillary defect. Goldmann applanation tonometry was 14 mmHg (normal) in both eyes. Anterior segment examination of the right eye was notable only for mild cataract, whereas the left eye had a posterior chamber intraocular lens. Dilated fundus examination of the right eye was notable for retinal pigment epithelial (RPE) changes with areas of geographic atrophy and pigment clumping in the macula. The left eye was notable for RPE changes and areas of macular geographic atrophy-similar to the right eye, but less pronounced.

\section{Diagnostic testing}

Fundus Autofluorescence (FAF) of both eyes (see Figure 3) demonstrated hypoautofluorescence in a peri-central ring-like distribution in the macula (a classic “bull's-eye pattern”), increased from the previous examination. Most notable in the left eye was marked hypoautofluorescent stippling in the peripapillary area closest to the optic nerve, extending from the superior vascular arcade to the inferior vascular arcade. 


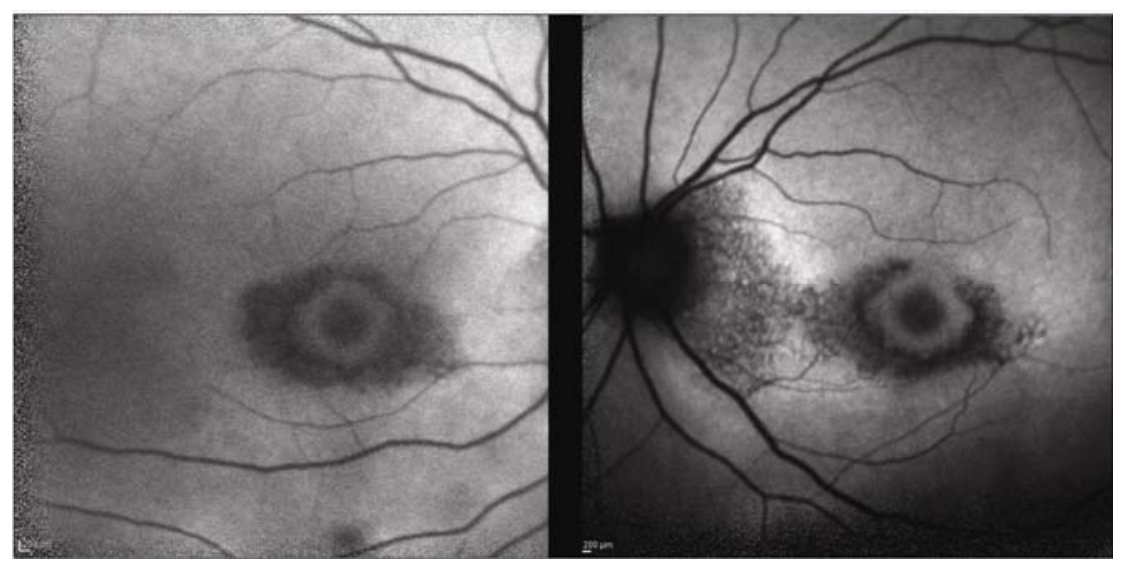

Figure 3. Fundus Autofluorescence of the right (left-most image) and the left (right-most image) eye from 2014 demonstrating advanced HCQ toxicity with marked RPE irregularities. Progression from prior studies and involvement of the nasal macula is now shown

Optical Coherence Tomography (OCT) of both eyes (see Figure 4) demonstrated disruption of the outer retina, RPE granularity, and perifoveal thinning of the inner and outer retinal layers, significantly progressed from earlier images. Both eyes display the "flying saucer sign," which represents relative sparing of the sub-foveal ellipsoid zone in the outer retina ${ }^{[1]}$.

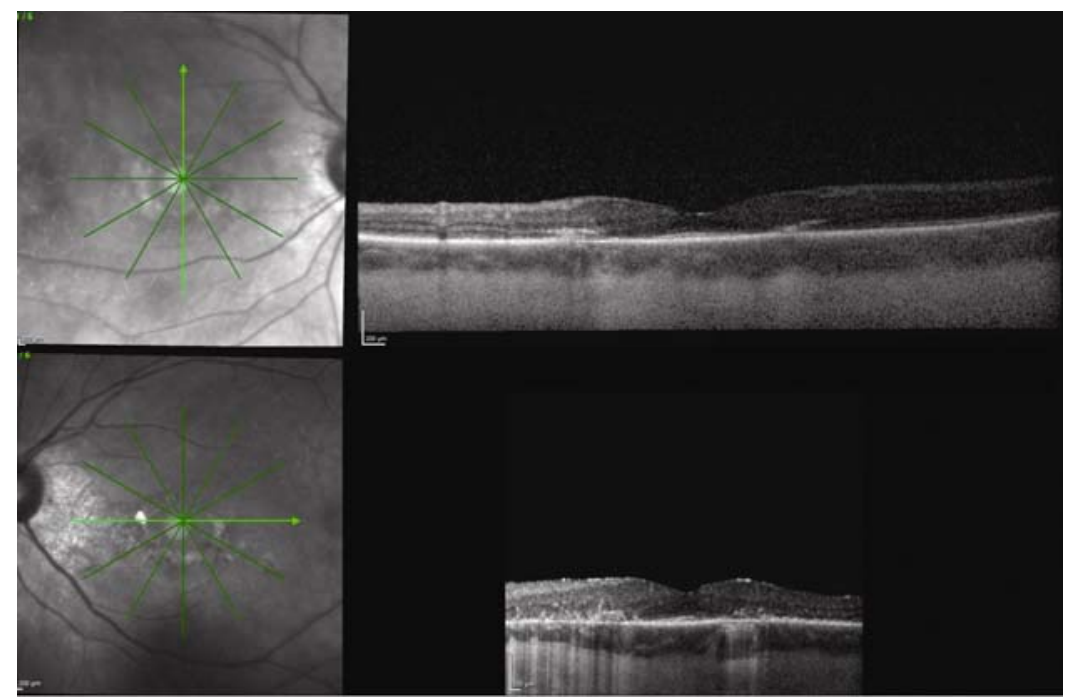

Figure 4. Optical Coherence Tomography of the right (upper image) and the left (lower image) eye from 2014 demonstrating more progressive disruption of the retinal layers, when compared to 2012

\section{Discussion}

Initially marketed as an anti-malarial drugs, chloroquine (CQ), and its analogue HCQ, are frequently used by physicians for long-term management of systemic lupus erythematous, rheumatoid arthritis, and other autoimmune conditions ${ }^{[2]}$. Retinopathy secondary to CQ toxicity is still a problem worldwide but is rarely seen in the United States because the drug has been essentially replaced by HCQ ${ }^{[3]}$. Although HCQ is taken by an estimated 150,000 people per year, the incidence of retinal toxicity is relatively rare, with rates ranging from $0.5 \%-7.5 \%$ in long-termusers ${ }^{[4-8]}$. Frustratingly, the mechanism of CQ and HCQ toxicity is not well elucidated. It is known that both drugs affect the metabolism of various 
retinal cells (including photoreceptors), but correlating these effects to the clinical picture, and to the areas of slow and chronic damage, have been more challenging ${ }^{[3]}$.

In early stages of toxicity, patients are commonly asymptomatic with preserved central vision, but may rarely detect difficulty with reading, night vision, and/or glare, or a symptomatic paracentral scotoma ${ }^{[9,10]}$. These subtle, subjective visual complaints are impossible to correlate with any early findings on clinical examination. Later on, clinical evidence of HCQ retinopathy can become apparent on fundus examination as a macular bull's-eye pattern, characterized by a ring of RPE degeneration often sparing the foveal center. The late onset findings and symptoms are irreversible and potentially progressive even with cessation of the drug. For these reasons, ophthalmoscopy alone is not an appropriate or effective screening mechanism ${ }^{[11]}$.

After initially publishing a Preferred Practice Pattern for HCQ Retinopathy screening in 2002, the American Academy of Ophthalmology (AAO) recently revised these criterion to include newer, more sensitive diagnostic imaging modalities ${ }^{[3,12,13]}$. The goal of careful screening is to detect early elements of retinal toxicity in attempt to avoid more advanced visual loss. Prior to initiating HCQ therapy, all patients are recommended to undergo a baseline ophthalmologic examination. If an individual is thought to be low risk for retinopathy, follow up examinations should be performed five years after the baseline exam. For those at higher risk of retinopathy-an individual with more than five years of HCQ use, more than 1,000 grams of cumulative consumption, more than $6.5 \mathrm{mg} / \mathrm{kg} /$ day dosing, increased age, concurrent hepatic/renal disease, or concomitant maculopathy of another etiology-annual follow up should begin immediately ${ }^{[3]}$. Each visit (baseline and follow-up) should include a detailed ophthalmologic examination with biomicroscopy and automated visual field testing, as well as one of the following additional, objective tests: 1) spectral-domain optical coherence tomography, 2) multifocal electroretinography, or 3) fundus autofluorescence.

Although most literature has emphasized that the risk of developing HCQ toxicity is linked to daily doses $>6.5 \mathrm{mg} / \mathrm{kg}$ of body weight, more recent surveys suggest that the total cumulative exposure to HCQ was far more significant ${ }^{[6,14,15]}$. Both in daily practice, and in the aforementioned surveys, patients typically receive $400 \mathrm{mg}$ of HCQ per day; too few individuals in these surveys received "higher-than-normal" or "unusual" dosages to significantly evaluate toxicity at these higher concentrations. Because of the large percentage of reported HCQ toxicity cases with patients who received HCQ at dosages $>6.5 \mathrm{mg} / \mathrm{kg}$, the current recommendation remains to dose the medication according to the patient's ideal body weight remaining in the range of $6.5 \mathrm{mg} / \mathrm{kg}$ daily ${ }^{[3]}$. Most often, a dose of $400 \mathrm{mg}$ of HCQ daily is appropriate, but special attention should be paid to those patients who are obese and those who are of short stature. Overdose of HCQ can occur for those of short stature where $400 \mathrm{mg}$ daily is too high a dose. Additionally, obese patients should be dosed based on their height (i.e., ideal body weight). HCQ distributes poorly in adipose tissues, so a dosing scale based on ideal body weight reduces the theoretical risk of overdosing overweight patients ${ }^{[3]}$.

At the time of her initial presentation two years ago, our patient already had early signs of toxicity; RPE changes in the macula and findings on FAF and OCT that were concerning for an advanced HCQ maculopathy. Although progression of the maculopathy was likely to continue even with immediate cessation of HCQ, her continued usage led to advanced stages of toxicity. The areas of hypoautofluorescence on FAF, which correspond to severely damaged or degenerated RPE cells, have increased, intensified, and are starting to involve areas of the nasal macula near the optic nerve. On OCT, the progression of retinal layer disruption has been marked, highlighted by a loss of the external limiting membrane, disruption of the outer ellipsoid zone, RPE granularity, and progressive parafoveal thinning of all retinal layers. As most patients in the United States undergo regular screenings and stop HCQ use at the first hint of toxicity, this is a rather rare and dramatic glimpse into what was previously a much more common occurrence. Even though she had been continuing HCQ against medical advice, every effort was made to re-educate her again on the urgency of stopping treatment.

Even though there are case reports where early or progressive HCQ toxicity has been documented ${ }^{[16]}$, this is a unique case where the patient continued the medication against medical advice and there is now documented progression of the retinal 
toxicity while still taking the medication. With some regularity, Ophthalmologists are faced with the difficult clinical dilemma to determine if a patient should discontinue HCQ therapy. This case emphasizes why detecting clinical toxicity and discontinuing therapy is crucial for the safe usage of this frequently prescribed medication.

\section{Conclusion}

HCQ is an extremely useful drug with a generally favorable side-effect profile. Although it occurs relatively infrequently, HCQ maculopathy is irreversible and must be detected early. Using the AAO's updated framework as a guide and operating with a low index of clinical suspicion, ophthalmologists can achieve early detection of HCQ maculopathy and help prevent severe visual loss.

\section{References}

[1] Chen E, Brown DM, Benz MS, et al. Spectral domain optical coherence tomography as an effective screening test for hydroxychloroquine retinopathy (the "flying saucer” sign). Clin Ophthalmol. 2010; 4: 1151-1158. http://dx.doi.org/10.2147/OPTH.S14257

[2] Mavrikakis M, Papazoglou S, Sfikakis PP, et al. Retinal toxicity in long term hydroxychloroquine treatment. Ann Rheum Dis. 1996; 55: 187-9. PMid:8712882 http://dx.doi.org/10.1136/ard.55.3.187

[3] Marmor MF, Kellner U, Lai TY, et al. Revised recommendations on screening for chloroquine and hydroxychloroquine retinopathy. Ophthalmology. 2011; 118: 415-422. PMid:21292109 http://dx.doi.org/10.1016/j.ophtha.2010.11.017

[4] Semmer AE, Lee MS, Harrison AR, et al. Hydroxychloroquine retinopathy screening. Br J Ophthalmol. 2008; 92(12): $1653-1655$. PMid:18829634 http://dx.doi.org/10.1136/bjo.2008.144402

[5] Mavrikakis I, Sfikakis PP, Mavrikakis E, et al. The incidence of irreversible retinal toxicity in patients treated with hydroxychloroquine: a reappraisal. Ophthalmology. 2003; 110: 1321-6. http://dx.doi.org/10.1016/S0161-6420(03)00409-3

[6] Wolfe F, Marmor MF. Rates and predictors of hydroxychloroquine retinal toxicity in patients with rheumatoid arthritis and systemic lupus erythematosus. Arthritis Care Res (Hoboken). 2010; 62(6): 775-84. PMid:20535788 http://dx.doi.org/10.1002/acr.20133

[7] Levy GD, Munz SJ, Paschal J, et al. Incidence of hydroxychloroquine retinopathy in 1,207 patients in a large multicenter outpatient practice. Arthritis Rheum. 1997; 40(8): 1482-1486. PMid:9259429 http://dx.doi.org/10.1002/art.1780400817

[8] Melles RB, Marmor MF. The risk of toxic retinopathy in patients on long-term hydroxychloroquine therapy. JAMA Ophthalmol. 2014; 132(12): 1453-1460. PMid:25275721 http://dx.doi.org/10.1001/jamaophthalmol.2014.3459

[9] Mititelu M, Wong BJ, Brenner M, et al. Progression of hydroxychloroquine toxic effects after drug therapy cessation: New evidence from multimodal imaging. JAMA Ophthalmol. 2013; 131: 1187-1197. PMid:23887202 http://dx.doi.org/10.1001/jamaophthalmol.2013.4244

[10] Michaelides M, Stover NB, Francis PJ, et al. Retinal toxicity associated with hydroxychloroquine and chloroquine: Risk factors, screening, and progression despite cessation of therapy. Arch Ophthalmol. 2011; 129: 30-39. PMid:21220626 http://dx.doi.org/10.1001/archophthalmol.2010.321

[11] Marmor MF. Comparison of screening procedures in hydroxychloroquine toxicity. Arch Ophthalmol. 2012; 130: $461-469$. PMid:22159170 http://dx.doi.org/10.1001/archophthalmol.2011.371

[12] Multimodal Imaging in Plaquenil Toxicity. Review of Ophthalmology Web site. http://www.reviewofophthalmology.com/content/d/retinal_insider/c/49802 Published August 6, 2014. Accessed January 23,2015.

[13] Marmor MF, Carr RE, Easterbrook M, et al. Recommendations on screening for chloroquine and hydroxychloroquine retinopathy: A report by the American Academy of Ophthalmology. Ophthalmology. 2002; 109: 1377-1382.

http://dx.doi.org/10.1016/S0161-6420(02)01168-5

[14] Lyons JS, Severns ML. Using multifocal ERG ring ratios to detect and follow Plaquenil retinal toxicity: a review: Review of mfERG ring ratios in Plaquenil toxicity. Doc Ophthalmol. 2009; 118: 29-36. PMid:18465156 http://dx.doi.org/10.1007/s10633-008-9130-0

[15] Maturi RK, Folk JC, Nichols B, et al. Hydroxychloroquine retinopathy. Arch Ophthalmol. 1999; 117: 1262-1263. PMid:10496407 http://dx.doi.org/10.1001/archopht.117.9.1262

[16] Phillips BN, Chun DW. Hydroxychloroquine retinopathy after short-term therapy. Retin Cases Brief Rep. 2014; 8(1): 67-9. PMid:25372212 http://dx.doi.org/10.1097/ICB.0000000000000006 